\title{
Small core Chalcogenide photonic crystal fiber for mid- infrared wavelength conversion: experiment and design
}

\author{
Sida Xing ${ }^{1}$, Davide Grassani ${ }^{1 *}$, Svyatoslav Kharitonov ${ }^{1}$, Adrien Billat ${ }^{1}$ and Camille Sophie Brès ${ }^{1}$ \\ Ecole Polytechnique Fédérale de Lausanne (EPFL), Photonic Systems Laboratory, STI-IEL, Station 11, CH-1015 Lausanne, Switzerland \\ *davide.grassani@epfl.ch
}

\begin{abstract}
Kerr index and dispersion parameter of a small core chalcogenide photonic crystal fiber are estimated via four-wave mixing near $2 \mu \mathrm{m}$. From these values, new fiber design is proposed to efficiently generate idlers in mid-infrared.

OCIS codes: (190.4370) Nonlinear optics, fibers; (190.4380) Nonlinear optics, four-wave mixing.
\end{abstract}

\section{Introduction}

The short-wave (SWIR) (1700-2500 nm) and the mid-infrared (MIR) are becoming key wavelength regions for a large number of applications, such as gas sensing [1], free space communication [2], medical diagnostic and surgery [3]. Moreover, laser sources around 2 micron can be employed for pumping nonlinear media to convert telecom signals in the MIR through four-wave mixing (FWM). Because of the excessive losses of silica beyond two micron, alternative materials exhibiting strong optical nonlinearity and large transparency windows in the MIR are under investigation. Beside silicon integrated platforms [4], promising all-fiber solutions are represented by microstructured chalcogenide glass $(\mathrm{ChG})$ optical fibers. Chalcogenide glasses show wide transparency windows in the MIR, and a very high third order nonlinearity, about 1000 times that of silica. In addition ChG photonic crystal fibers (PCF) allow dispersion engineering and long interaction length with very low propagation losses. Inside ChG fibers, FWM have been demonstrated in the telecom band [5], together with Raman scattering at $2 \mu \mathrm{m} \mathrm{[6]} \mathrm{and}$ supercontinuum generation in the MIR [7]. Recently we observed FWM in the 2 micron band in an AsSe PCF [8]. However, due to material constraints, the PCF had a large core diameter of 14 micron, so that material dispersion strongly dominated leading to a zero dispersion wavelength (ZDW) near 5.1 micron.

Here we show FWM experiment in a GeAsSe PCF in the 2 um region. Doping the ChG glass with germanium enabled the fabrication of a smaller core fiber ( 4 micron diameter) and stronger waveguide dispersion. FWM is demonstrated with continuous wave (CW) pump, which is highly desirable for spectroscopic and optical communication applications. From the derived dispersion and Kerr index we propose a new realistic design for a tapered GeAsSe PCF showing a potential increase of the conversion efficiency (CE) and an extension of the conversion bandwidth further into SWIR and MIR regions.

\section{Experimental setup and results}

Fig.1 shows the experimental set-up. The fiber under test (FUT) is a $27.5 \mathrm{~cm}$ long $\mathrm{Ge}_{10} \mathrm{As}_{22} \mathrm{Se}_{68}$ PCF with core size of $4 \mu \mathrm{m}$ and diameter-to-pitch ratio $r=0.49$ fabricated by PERFOS. Pump and the signal lasers are two custommade continuous wave (CW) thulium (Tm)-doped fiber laser [9]. The pump wavelength is fixed at $1980.6 \mathrm{~nm}$ and has a linewidth of $0.2 \mathrm{~nm}$. The signal laser is tunable over $1950 \mathrm{~nm}-2050 \mathrm{~nm}$ range and has a linewidth of about 0.4 $\mathrm{nm}$. Light is coupled in and collected from the FUT by two lensed fibers with a spot size diameter of $4 \mu \mathrm{m}$ at $2 \mu \mathrm{m}$ wavelength. Input and output losses are about $4 \mathrm{~dB}$ and we estimated propagation losses to be about $2 \mathrm{~dB} / \mathrm{m}$. Finally, the FUT's output is sent to an optical spectrum analyzer for data recording and analysis.

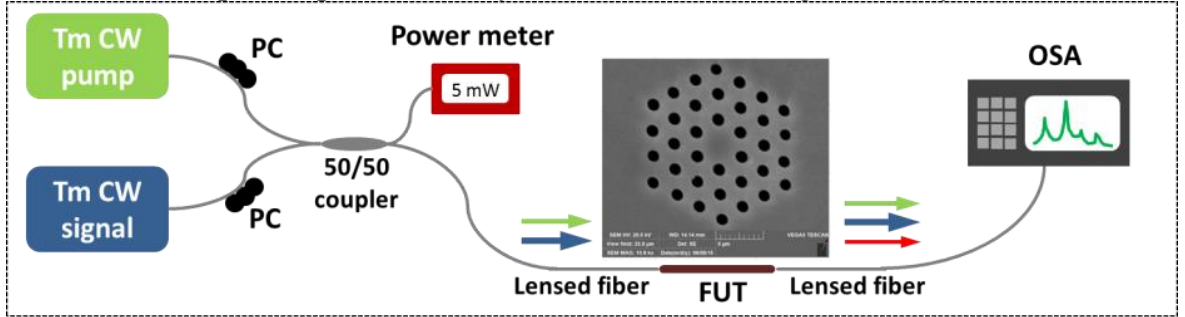

Fig. 1 Experimental FWM set-up. OSA: optical spectrum analyzer; PC: polarization controller. Fiber's SEM image provided by PERFOS.

In Fig.2a, we show the experimental CE, defined as output idler power over input signal power, as a function of idler wavelength. The coupled pump power is $65 \mathrm{~mW}$ and the signal was swept from $1983 \mathrm{~nm}$ to $2015 \mathrm{~nm}$. A good $\mathrm{CE}$ of $-30 \mathrm{~dB}$ is obtained over $20 \mathrm{~nm}$ of total bandwidth. The dispersion estimated from the conversion bandwidth is $\mathrm{D}=-183 \mathrm{ps} /(\mathrm{nm} \mathrm{km})$, and compares well with the value $\mathrm{D}=-196 \mathrm{ps} /(\mathrm{nm} \mathrm{km})$ retrieved from COMSOL simulations, 
where we used the actual fiber geometry and Sellmeier equation for $\mathrm{Ge}_{11.5} \mathrm{As}_{24} \mathrm{Se}_{64.5}$ [10]. Fig.2b shows $\mathrm{CE}$ values at different pump power levels for a $6 \mathrm{~nm}$ pump-signal detuning. The CE increases quadratically with the pump power showing a maximum $\mathrm{CE}=-25 \mathrm{~dB}$ with no sign of saturation. A nonlinear coefficient $\gamma$ of $1.688 \pm 0.11 \mathrm{~W}^{-1} \mathrm{~m}^{-1}$ is retrieved from the fitting of the experimental data in Fig.2b. Using the effective area $\left(\mathrm{A}_{\text {eff }}\right)$ computed from simulations, we estimated a Kerr index $\mathrm{n}_{2} \approx 2.65 \cdot 10^{-14} \mathrm{~cm}^{2} / \mathrm{W}$, in agreement with values reported in the literature [11]. The estimated ZDW is at $3.1 \mu \mathrm{m}$ (fig.3a), still too red shifted with respect to our pumping wavelength.
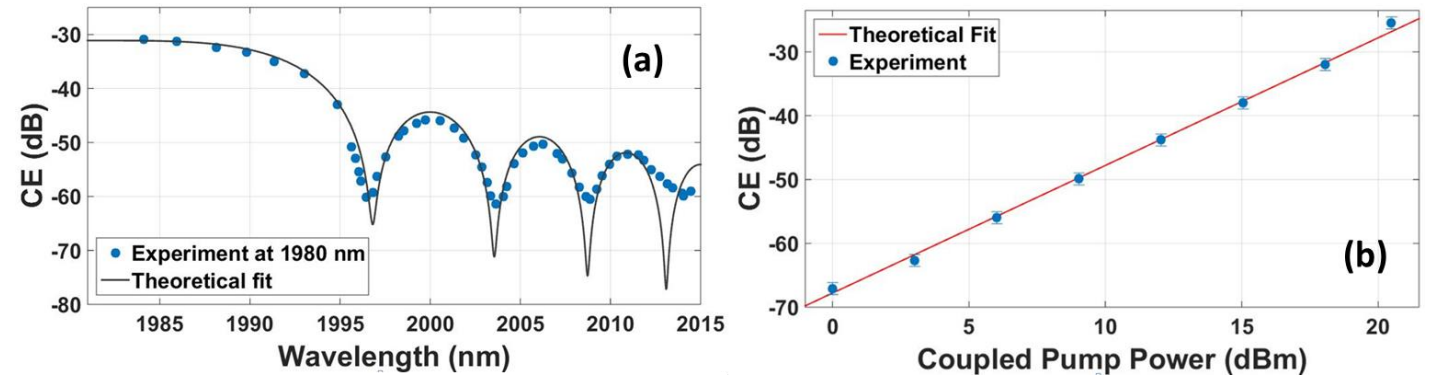

Fig. 2 (a) Experimental points superimposed to theoretical fits of the $\mathrm{CE}$ as a function of the idler wavelength; (b) $\mathrm{CE}$ as a function of the coupled pump power. Error bars are calculated considering $0.5 \mathrm{~dB}$ uncertainty on the input lasers power.

\section{Design of tapered GeAsSe PCF}

Having validated our simulations with the experimentally retrieved dispersion, we studied a new PCF geometry in order to further blue-shift the ZDW in the $2 \mu \mathrm{m}$ region, enhancing both CE and conversion bandwidth. For this purpose, we increased the $r$ value to 0.66 and decreased the core diameter to $1.5 \mu \mathrm{m}$ by assuming a tapered geometry [11]. Both of these values represent the current state of the art in the fabrication process. With this geometry, the ZDW was estimated to be around $1.967 \mu \mathrm{m}$ (fig.3a). In our case, it should be noted that the main purpose of tapering lies in dispersion engineering and not in nonlinearity enhancement.
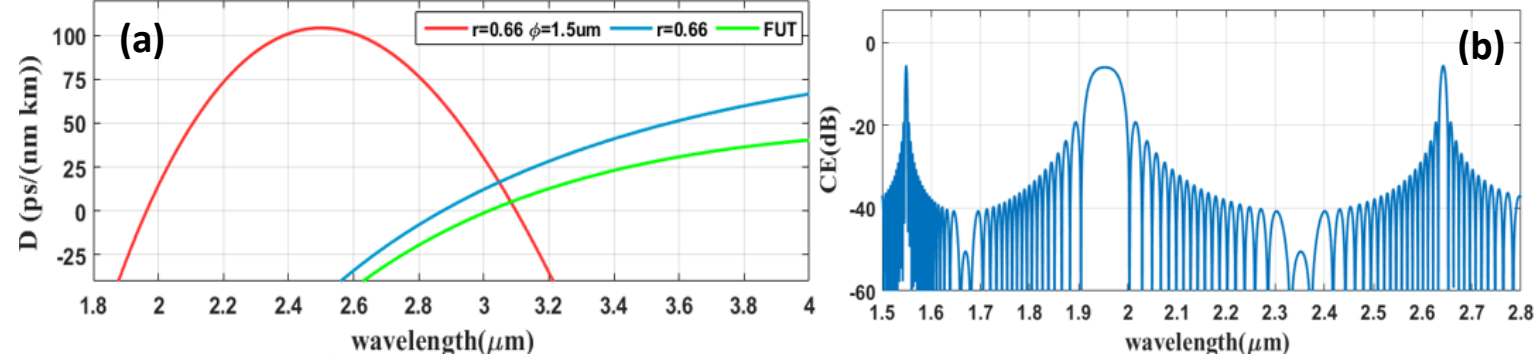

Fig. 3(a) Simulated dispersion for FUT (green), tapered (red), and un-tapered (blue) fiber.(b) Simulated CE in the taper with pump at $1.953 \mu \mathrm{m}$.

The theoretical CE was then simulated for a $1 \mathrm{~m}$ length tapered region, considering a pump power of $50 \mathrm{~mW}$ at $1.953 \mu \mathrm{m}$, in the maximum emission region of Tm doped fiber lasers. A maximum CE of $-6 \mathrm{~dB}$ is found and the $3 \mathrm{~dB}$ bandwidth around the pump is about $60 \mathrm{~nm}$. Linear losses should increase by less than $1 \mathrm{~dB} / \mathrm{m}$ in the tapered region [11], thus a slightly lower CE is experimentally expected. An additional interesting feature is also observed since wavelength conversion of telecom signal at $1.55 \mu \mathrm{m}$ to the mid-IR range at $2.643 \mu \mathrm{m}$ is expected (fig. $3 \mathrm{~b}$ ).

In conclusion, we demonstrated FWM at $2 \mu \mathrm{m}$ at low pumping power in $\mathrm{CW}$ regime in $27.5 \mathrm{~cm}$ long $\mathrm{ChG}$ PCF. Excellent match with theory confirms the good uniformity of the fiber. We numerically showed that by state-of-theart fabrication techniques, larger CE, bandwidth and distant conversion can be achievable with the same material. This work was supported by the European Research Council under grant agreement ERC-2012-StG 306630-MATISSE. We acknowledge Laurent Brilland and Johann Troles for fruitful discussions.

[1] S. Moro, et al., "Widely-tunable parametric short-wave infrared transmitter for CO 2 trace detection," Optics express 19, 8173 (2011).

[2] C. Weitkamp, Lidar: range-resolved optical remote sensing of the atmosphere (Springer, 2006), Vol. 102.

[3] R. Anderson, "Selective photothermolysis of lipid-rich tissues: A free electron laser study," Lasers in Surgery and Medicine 38, 913 (2006).

[4] X. Liu, et al., "Mid-infrared optical parametric amplifier using silicon nanophotonic waveguides," Nature Photonics 4, 557 (2010).

[5] C.-S. Brès, et al., "Continuous-wave four-wave mixing in cm-long Chalcogenide microstructured fiber," Optics express 19, B621 (2011).

[6] J. Troles, et al., "Low loss microstructured chalcogenide fibers for large non linear effects at 1995 nm," Optics Express 18, 26647 (2010).

[7] C. R. Petersen, et al., "Mid-infrared supercontinuum covering the 1.4-13.3 $\mu \mathrm{m}$ molecular fingerprint region using ultra-high NA chalcogenide step-index fibre," Nature Photonics 8, 830 (2014).

[8] D. Grassani, et al., "Continuous wave four-wave mixing at 2 micron in Chalcogenide microstructured fiber," in Advanced Solid State Lasers, , ATu3A. 6.2015

[9] S. Kharitonov, et al., "Kerr nonlinearity of Thulium-doped fiber near $2 \mu \mathrm{m}, "$ in CLEO: Science and Innovations, JTu5A. 31.

[10] P. Ma, et al., "Low-loss chalcogenide waveguides for chemical sensing in the mid-infrared," Optics Express 21, 29927 (2013)

[11] S. D. Le, et al., "Wavelength conversion in a highly nonlinear chalcogenide microstructured fiber," Optics Letters 37, 4576 (2012). 